\title{
Real-Time Smart Patient Monitoring and Assessment Amid COVID-19 Pandemic - an Alternative Approach to Remote Monitoring
}

\author{
B Naveen Naik ${ }^{1} \cdot$ Rekha Gupta $^{1} \cdot$ Ajay Singh $^{1}$ (D) Shiv Lal Soni ${ }^{1} \cdot$ G D Puri ${ }^{1}$
}

Received: 25 May 2020 / Accepted: 4 June 2020 / Published online: 13 June 2020

(C) Springer Science+Business Media, LLC, part of Springer Nature 2020

Sir,

The largest-ever COVID-19 outbreak is ravaging the world. This highly transmissible viral infection has led to thousands of confirmed, probable, or suspected cases in India so far, increasing burden on the health care system. Despite no proven COVID-19 specific medical therapy, with basic monitoring and supportive treatment, many of the infected patients do not need to die. COVID-19 represents an illness ready for a paradigm shift in the health care delivery and outcome, challenging the intensivists to ensure it happened. Tele-ICU technology with remote consultation is seen as a way to help hospitals cope with fewer intensivists and staff. This technology contributes to a $15-60 \%$ reduction in mortality and a $30 \%$ reduction in the average length of stay $[1,2]$. Tele-ICU appears to be a promising path in the present era, but its adoption requires a huge capital investment which hinders its implementation. An estimation of $\$ 2-5$ million is probably the cost to set up a command centre and install the tele- ICU systems, with an operating cost of $\$ 600,000-1.5$ million per year is reported from different adaptors [3].

This article is part of the Topical Collection on Mobile \& Wireless Health

Ajay Singh

ajay.ydv2509@gmail.com

B Naveen Naik

navin_amc@yahoo.com

Rekha Gupta

guptarekha19.rg@gmail.com

Shiv Lal Soni

dr.shivsoni@gmail.com

G D Puri

gdpuri007@hotmail.com

1 Department of Anaesthesia \& Intensive Care, Post Graduate Institute of Medical Education and Research, Sector 12, Chandigarh 160012, India
Facing the daunting outlook of COVID-19 pandemic surge, with limited ICU beds, resources and health care personnel, it is imperative to safely monitor and care for sickest patients. Impending crisis are opportunities for innovation, wherein a normally slow-moving healthcare system can be improvised and innovated in response to the pandemic. Utilization of remote technology to manage a deluge of critically ill COVID-19 patients may ease the burden on health care facilities and can stem the exposure of health care providers to COVID-19. But its use must be tempered with a focus on data privacy and cybersecurity. Providing better patient care services round the clock is of utmost importance. We implemented a technology for remote monitoring of ICU, utilizing closed-circuit television (CCTV) cameras and smartphones. High definition CCTV cameras were installed over each ICU bed for visualising patient mechanical ventilation and monitoring system round the clock (Fig. 1b). We have reported a similar CCTV system with audio-visual communication in the doffing area to enhance staff safety, monitored from remote location [4]. In addition to this, we installed a remote mobile health monitoring system and server structure, where patient vital parameters such as temperature, $\mathrm{SpO} 2, \mathrm{ECG}$, heart rate, blood pressure, etCO2 and respiratory rate can be visualized over smartphone from the off-site location (Fig. 1a). It assists the skilled intensivist to monitor, alert and manage patient care without being physically present bedside [5]. Remote ICU surveillance technology amid COVID-19 pandemic has the following benefits:

- Flexible, agile and more economical system

- Allows experienced intensivists to remotely monitor the status of many patients in ICU over a smartphone.

- Ensures continuous vital physiological monitoring round the clock.

- Sudden clinical deterioration can be detected earlier and onsite clinicians can be alerted to intervene timely. 
Fig. 1 Remote patient monitoring using (a) Smartphone and (b) Closed-circuit television (CCTV)

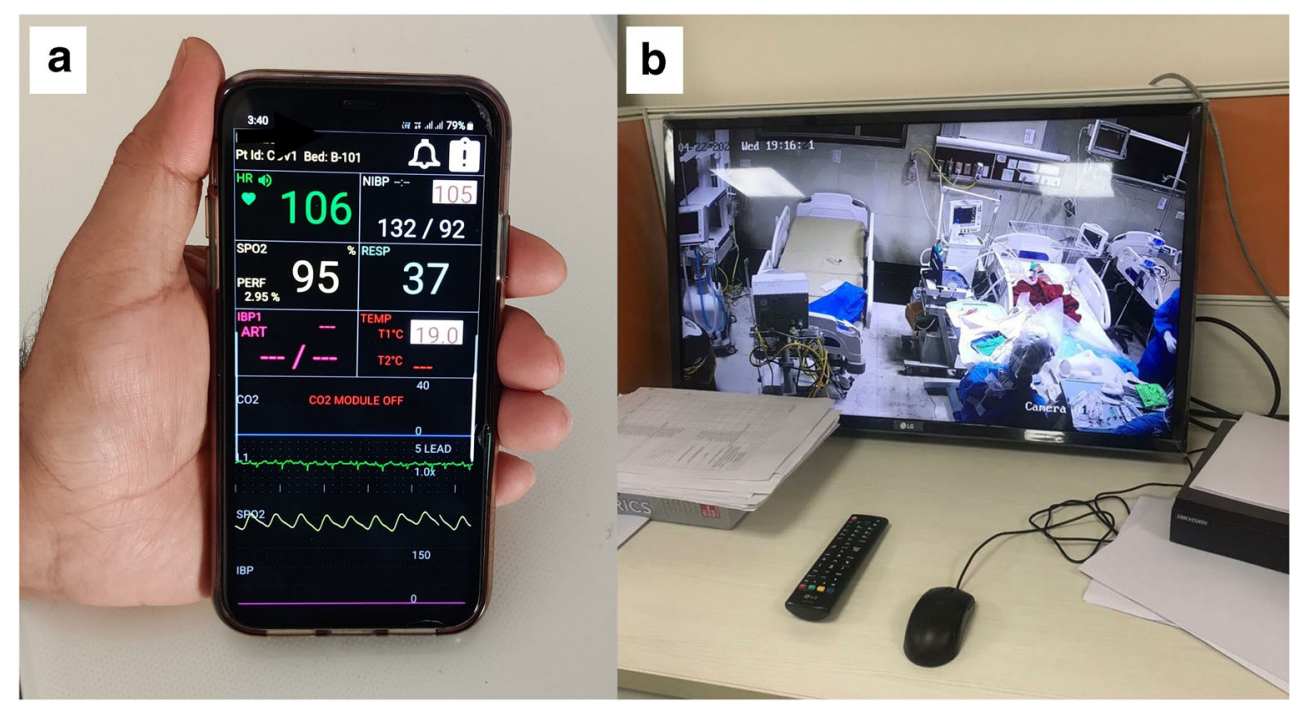

- Ensures quality health care with limited resources and personnel.

- Allows quarantined intensivists to monitor from an offsite location.

Acknowledgements Not applicable.

Source of Funding Not applicable.

Authors Contribution B Naveen Naik: This author helped with concept, design, manuscript preparation and editing.

Rekha Gupta: This author helped with literature search, manuscript preparation and editing.

Ajay Singh: This author helped with manuscript preparation and editing.

Shiv Lal Soni: This author helped with manuscript editing and manuscript review.

G D Puri: This author helped with manuscript editing and manuscript review.

\section{Compliance with Ethical Standards}

Conflict of Interest All authors report no conflicts of interest to declare.

\section{References}

1. Kumar S, Merchant S, Reynolds R. Tele-ICU: efficacy and costeffectiveness of remotely managing critical care. Perspect Health Inf Manag 2013; 10:1

2. Rosenfeld BA, Dorman T, Breslow MJ, et al. Intensive care unit telemedicine: Alternate paradigm for providing continuous intensivist care. Crit Care Med 2000; 28(12): 3925-31

3. Breslow MJ, Rosenfeld BA, Doerfler M, Burke G, Yates G, Stone DJ et al. Effect of a multiple-site intensive care unit telemedicine program on clinical and economic outcomes: An alternative paradigm for intensivist staffing. Crit Care Med 2004; 32(1): 31-8.

4. Singh A, Naik BN, Soni SL, Puri GD. Real-Time Remote Surveillance of Doffing during COVID-19 Pandemic: Enhancing Safety of Health Care Workers. Anesth Analg. 2020 May 1. doi: https://doi.org/10.1213/ANE.0000000000004940. [Epub ahead of print] PubMed PMID

5. Zhang Y, Liu H, Su X, Jiang P, Wei D. Remote Mobile Health Monitoring System Based on Smart Phone and Browser/Server Structure. J Healthc Eng. 2015;6(4):717-737.

Publisher's Note Springer Nature remains neutral with regard to jurisdictional claims in published maps and institutional affiliations. 